\title{
Investigation of the Effects of Intra-articular Tranexamic Acid on Intact Cartilage Tissue and Cartilage Formation in Osteochondral Defects of the Rabbit Knee: An Experimental Study
}

\author{
Fevzì Bìrìsik Sr. ${ }^{1}$, Serkan Bayram ${ }^{2}$, Mehmet Çakmak ${ }^{3}$, Evşen Apaydın ${ }^{4}$, Ali Erşen ${ }^{2}$ \\ 1. Orthopaedics and Traumatology, Istanbul Training and Research Hospital, Istanbul, TUR 2. Orthopaedics and \\ Traumatology, Istanbul University School of Medicine, Istanbul, TUR 3. Orthopaedics and Traumatology, University \\ College of Medical Sciences, Kirsehir, TUR 4. Pathology, Istanbul University School of Medicine, Istanbul, TUR
}

Corresponding author: Fevzi Bìrisik Sr., dr.fevzibirisik@gmail.com

\section{Abstract \\ Background}

In this study, the effects of tranexamic acid (TXA) and saline on intact cartilage and the recovery of experimental osteochondral lesions following microfracture in a rabbit model were compared.

\section{Methods}

Twenty adult rabbits were divided into four groups (1A, 1B, 2A, and 2B) based on with or without TXA use and microfracture. In addition, these groups were categorized into two different subgroups based on the use of TXA in Groups 1 and 2 (Groups A and B). Full-thickness cartilage defects were created on the weightbearing surface of the medial femoral condyles unilaterally in Group 2 for the effect of TXA or saline on healthy cartilage tissue while a repetitive injection was applied in Group 1 for the effect of TXA or saline on intact cartilage. A single dose of $10 \mathrm{mg} / \mathrm{kg}$ TXA was injected into the knee joints of Group A and $10 \mathrm{mg} / \mathrm{kg}$ $0.9 \%$ saline solution injected in Group B for three consecutive days. All animals were sacrificed for the extraction of the medial condyles for histologic evaluation eight weeks after surgery. The International Cartilage Repair Society (ICRS) II scoring system was used for histologic evaluation.

\section{Results}

No complications or adverse effects related to surgery were observed in all rabbits. All ICRS II parameters were similar in the TXA and saline solution groups in the intact cartilage group except for chondrocyte clustering, formation of a tidemark, subchondral bone abnormalities, and mid/deep zone assessment. Moreover, these parameters were higher in the saline solution group in the cartilage group, but no significant difference was observed in the TXA group in the intact cartilage group. All ICRS II parameters were higher in the saline solution group than in the TXA group in the microfracture group, but no significant

Review began 04/23/2021 Review ended 05/02/2021 Published 05/06/2021

\section{๑) Copyright 2021}

Birisik et al. This is an open access article distributed under the terms of the Creative Commons Attribution License CC-BY 4.0., which permits unrestricted use, distribution, and reproduction in any medium, provided the original author and source are credited. difference was observed in the TXA group in the microfracture group except for inflammation, which was similar in the TXA and saline solution groups in the microfracture group.

\section{Conclusion}

We found that intra-articular TXA administration did not have a negative impact on healthy cartilage tissue and cartilage transformation and proliferation as compared to the saline infusion.

Categories: Pathology, Orthopedics, Quality Improvement

Keywords: tranexamic acid, osteochondral defects, intact cartilage, international cartilage repair society

\section{Introduction}

Tranexamic acid (TXA) is an antifibrinolytic agent that prevents plasmin formation, thus inhibiting the breakdown of fibrin clots [1-2]. Previous studies have reported the systemic and topical use of TXA for reducing blood loss and the need for blood transfusions [3-4]. Although TXA is used in major surgeries when higher blood loss is expected, such as hip arthroplasty [5], it may also be expansively used in minimally invasive procedures, such as knee arthroscopy, to improve the surgical approach and functional outcome by reducing postoperative bleeding and swelling [6]. TXA can be administered intravenously, intra-articularly, or orally in the setting of surgery. Although intravenous TXA is widely used, it may also be associated with postoperative seizures and increased thromboembolic events [7]. Therefore, intra-articular application is preferred for reducing blood loss and the need for blood transfusions, which has been increasing over the past several years [8].

Although the intra-articular application of TXA is commonly practiced due to its lower rates of systemic 
effects, the potential damage on articular cartilage following its intra-articular use is yet to be investigated. Tuttle et al. reported that TXA was cytotoxic to bovine and murine cartilage at the dose of $100 \mathrm{mg} / \mathrm{mL}$, but the safety cutoff dose was found to be $25 \mathrm{mg} / \mathrm{ml}$ for TXA [9-10]. However, no study has compared the effect of the intra-articular application of TXA on intact cartilage tissue and cartilage formation in osteochondral defects.

In this study, the effects of TXA and saline on intact cartilage and the recovery of experimental osteochondral lesions following microfracture in a rabbit model were compared.

\section{Materials And Methods}

Twenty-four male, adult, white New Zealand rabbits (18-24 months old and 3-3.50 kg) were provided by Istanbul University Aziz Sancar Institute of Experimental Medicine Laboratories (Istanbul, Turkey) and were maintained and housed at the same center of Istanbul University in accordance with the regulations set forth by the Office of Protection of Research Subjects. The study was performed under a protocol approved by the Istanbul University Istanbul Faculty of Medicine Animal Research Committee (2017/20385).

\section{Study design and surgical procedure}

Twenty adult rabbits were randomly divided into two groups based on whether they were with or without a microfracture (Groups 1 and 2). In addition, these groups were categorized into two different subgroups based on the use of TXA in Groups 1 and 2 (Group 1-A and 1-B; Group 2-A and 2-B) (Figure-1).

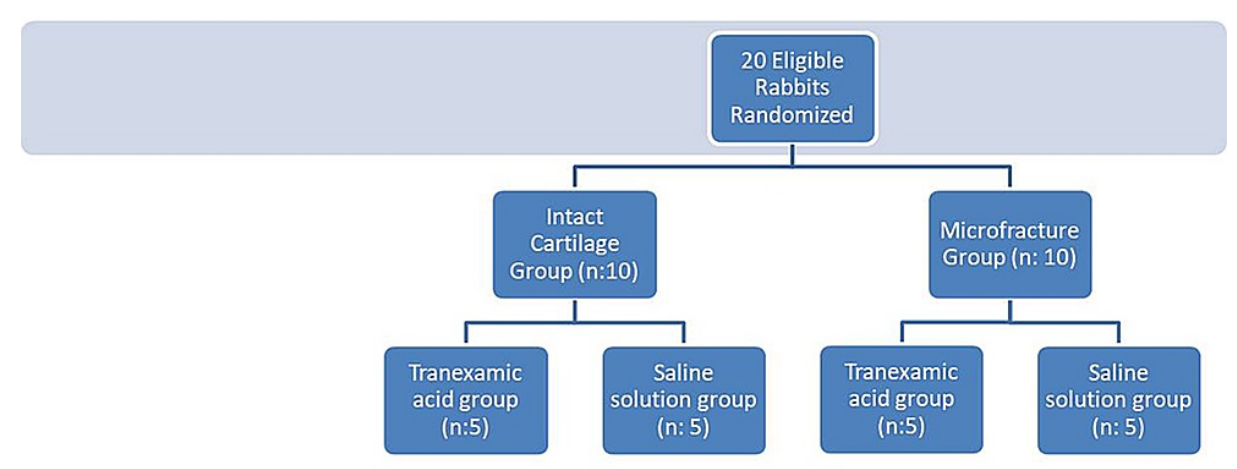

\section{FIGURE 1: Flow chart of the groups}

All rabbits were treated unilaterally in Group 2. A single orthopedic surgeon with previous experience in animal studies performed all the procedures. The rabbits were premedicated with buprenorphine 30 minutes before the procedure. The rabbits were anesthetized using $1 \mathrm{~mL}$ of xylazine and 1.0-1.2 $\mathrm{mL}$ of ketamine intramuscularly. Anesthesia was maintained with $1 \%-4 \%$ isoflurane. An aseptic technique was used for all surgical procedures. Preoperatively, all animals received antibiotic prophylaxis $(50 \mathrm{mg} / \mathrm{kg}$ cephalosporin sodium) subcutaneously. The right knees of the rabbits were shaved, and the surgical area was cleared of the hair, cleaned with betadine or alcohol, and closed with surgical dressing. A medial parapatellar arthrotomy was performed after the standard anterior midline skin incision, and the patella was everted laterally. Cylindrical osteochondral defects were created at the weight-bearing surfaces of the medial condyles of the femur, with a size of $4 \mathrm{~mm}$ wide and $2 \mathrm{~mm}$ deep, by using a dermal biopsy punch (Figure 2). 


\section{Cureus}

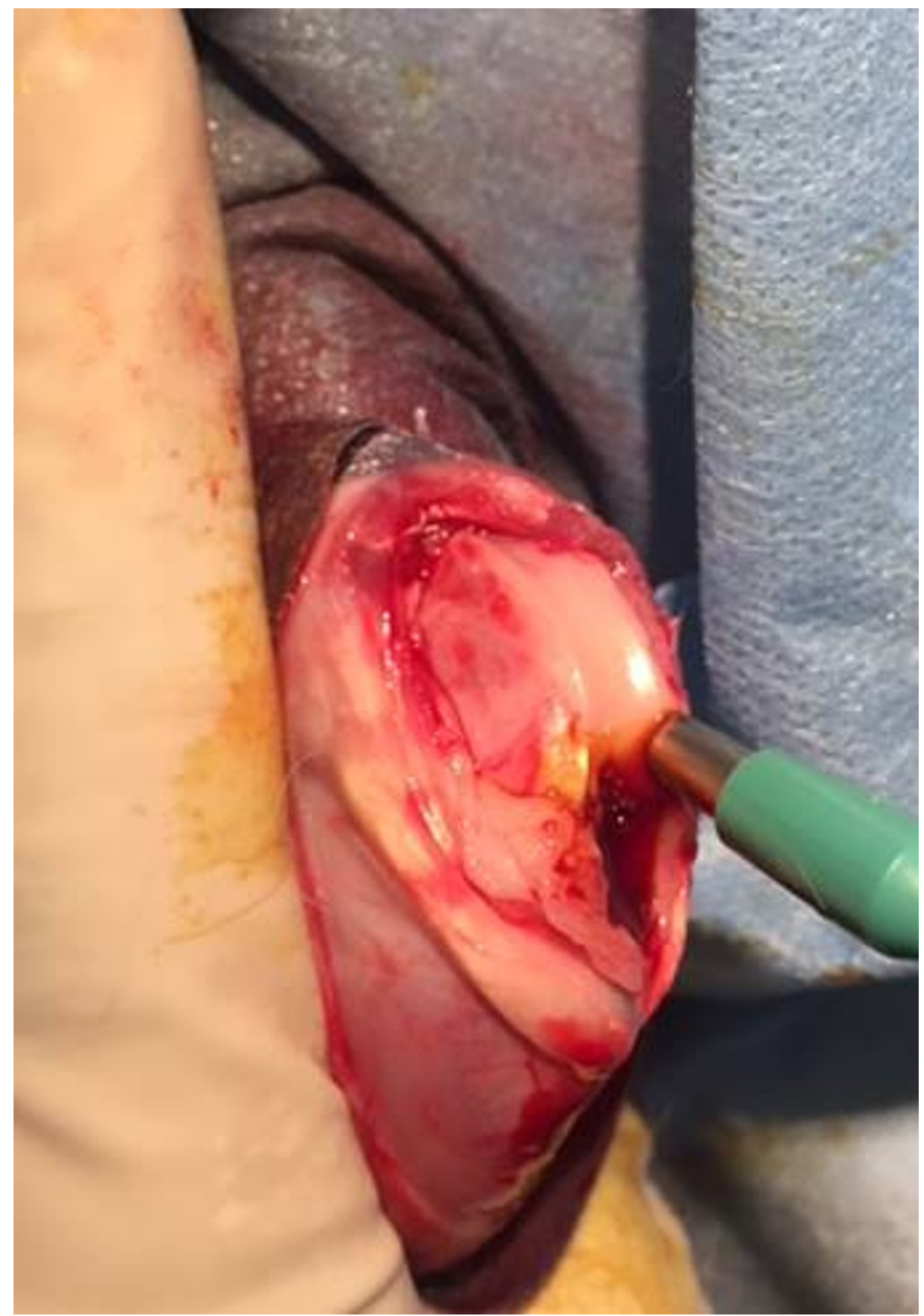

FIGURE 2: Cylindrical osteochondral defects were created at the weightbearing surfaces of the medial condyles of the femur, with a size of 4 $\mathrm{mm}$ wide and $2 \mathrm{~mm}$ deep, by using a dermal biopsy punch

The guidelines of the American Society for Testing and Materials International indicate that the critical size of a chondral defect in a rabbit is a diameter of $3 \mathrm{~mm}$ [11]. Watertight closure of the joint capsule was done with 3-0 Vicryl ${ }^{\circledR}$ and skin closure was applied with 3-0 Monocryl ${ }^{\circledR}$. Following the closure of the joint capsule, a single dose of $10 \mathrm{mg} / \mathrm{kg}$ TXA was injected into the knee joints of Group A and $10 \mathrm{mg} / \mathrm{kg} 0.9 \%$ saline solution injected in Group B for three consecutive days. In Group 1, a single dose of $10 \mathrm{mg} / \mathrm{kg}$ TXA was intraarticularly injected in Group A and $10 \mathrm{mg} / \mathrm{kg}$ 0.9\% saline solution in Group B for three consecutive days. All animals received antibiotic prophylaxis (cephalosporin sodium) postoperatively for one day. All rabbits were administered with postoperative non-steroid anti-inflammatory drugs for three to five days as required. The animals were maintained at a temperature of $20^{\circ} \mathrm{C} \pm 3^{\circ} \mathrm{C}$ with a relative humidity of $40 \%-60 \%$ and a photoperiod of $12 / 12 \mathrm{~h}$ (light and dark). All the rabbits were allowed to move freely and housed in a stainless steel cage with a bottom grid. The animals were fed with a pellet diet and had continuous access to tap water. 
Furthermore, all animals were sacrificed in accordance with the Institutional Animal Care and Use Committee Protocol with high-dose sodium pentobarbital (>200 $\mathrm{mg} / \mathrm{kg}$ ) for the extraction of the medial condyles for histologic evaluation eight weeks after surgery.

\section{Historical evaluation}

A macroscopic examination of the implantation site was performed by observing the appearance of the tissue in situ and photographic documentation. The acquired specimens of the distal femur were embedded in $10 \%$ formalin for one week and decalcified in $10 \%$ ethylenediaminetetraacetic acid (EDTA)-buffered saline solution for five days. Moreover, the samples were embedded in paraffin blocks and cut into $4 \mu \mathrm{m}$ sections. For histopathological evaluation, hematoxylin-eosin staining was used for the evaluation of the general morphology and safranin $\mathrm{O}$ for the evaluation of proteoglycan content. All sections were blindly scored by a single pathologist according to the International Cartilage Repair Society (ICRS) II scoring system, which had 14 grading criteria [12].

The ICRS II scoring system consists of 14 parameters, including tissue morphology (viewed under polarized light), matrix staining (metachromasia), cell morphology, chondrocyte clustering (four or more grouped cells), surface architecture, basal integration, formation of a tidemark, subchondral bone abnormalities/marrow fibrosis, inflammation, abnormal calcification/ossification, vascularization (within the repaired tissue), surface/superficial assessment, mid/deep zone assessment, and overall assessment. Each ICRS II parameter can range from 0 to 100. A higher score indicates better quality cartilage. Hence, a score of 0 was assigned for properties considered indicative of poor quality cartilage and 100 for goodquality cartilage. All parameters were scored with this method.

\section{Statistical analysis}

All statistical analyses were conducted using the Statistical Package for Social Sciences (SPSS ${ }^{\circledR}$ ) version 22.0 software (IBM Corp, Armonk, NY). Descriptive statistical methods were used to evaluate study data. The normality of distribution was tested using the Shapiro-Wilk test. Non-normally distributed variables were compared using the Mann-Whitney U test. Moreover, a P-value of $<0.05$ was regarded as significant.

\section{Results}

No complications or adverse effects related to surgery were observed in all rabbits. The macroscopic evaluation of femoral condyles showed that the defect areas in groups were filled with repair tissue, which is irregular and rough in varying proportions.

All ICRS II parameters were similar in the TXA and saline solution groups in the intact cartilage group except for chondrocyte clustering, formation of a tidemark, subchondral bone abnormalities, and mid/deep zone assessment. Moreover, these parameters were higher in the saline solution group in the cartilage group, but no significant difference was observed in the TXA group in the intact cartilage group (Table 1; Figures 3-4). 


\section{Cureus}

\begin{tabular}{|c|c|c|c|c|c|}
\hline \multirow[t]{2}{*}{ Parameters } & \multicolumn{2}{|c|}{ Tranexamic Acid Group (Group 1-A) } & \multicolumn{2}{|c|}{ Saline Solution Group (Group 1-B) } & \multirow[b]{2}{*}{$\mathrm{p}$ value } \\
\hline & Mean \pm SD & Min-Max & Mean \pm SD & Min-Max & \\
\hline Tissue morphology & 100 & & 100 & & 1 \\
\hline Matrix staining & 100 & & 100 & & 1 \\
\hline Cell morphology & 100 & & 100 & & 1 \\
\hline Chondrocyte clustering & $94 \pm 8$ & $80-100$ & $96 \pm 4.8$ & $90-100$ & 0.796 \\
\hline Surface architecture & 100 & & 100 & & 1 \\
\hline Basal integration & 100 & & 100 & & 1 \\
\hline Formation of a tidemark & $92 \pm 11.6$ & $70-100$ & $96 \pm 8$ & $80-100$ & 0.475 \\
\hline Subchondral bone abnormalities & $94 \pm 8$ & $80-100$ & $94 \pm 4.8$ & $90-100$ & 0.734 \\
\hline Inflammation & 100 & & 100 & & 1 \\
\hline Abnormal calcification/ossification & 100 & & 100 & & 1 \\
\hline Vascularization & 100 & & 100 & & 1 \\
\hline Surface/superficial assessment & 100 & & 100 & & 1 \\
\hline Mid/deep zone assessment & $94 \pm 4.8$ & $90-100$ & $98 \pm 4$ & $90-100$ & 0.394 \\
\hline Overall assessment & 100 & & 100 & & 1 \\
\hline
\end{tabular}

\section{TABLE 1: Comparison of ICRS II scoring between the intact cartilage groups}

ICRS: International Cartilage Repair Society

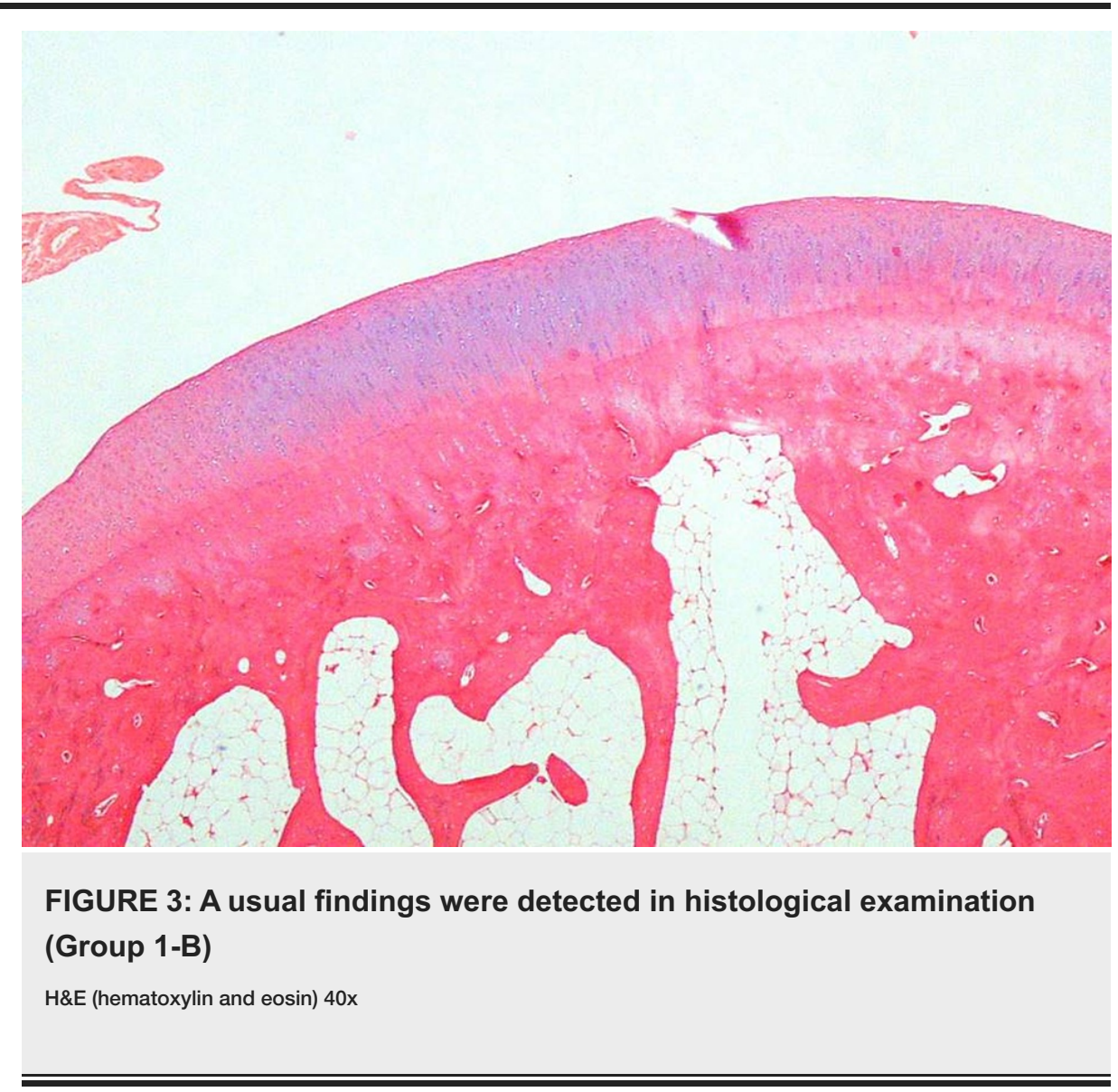




\section{Cureus}

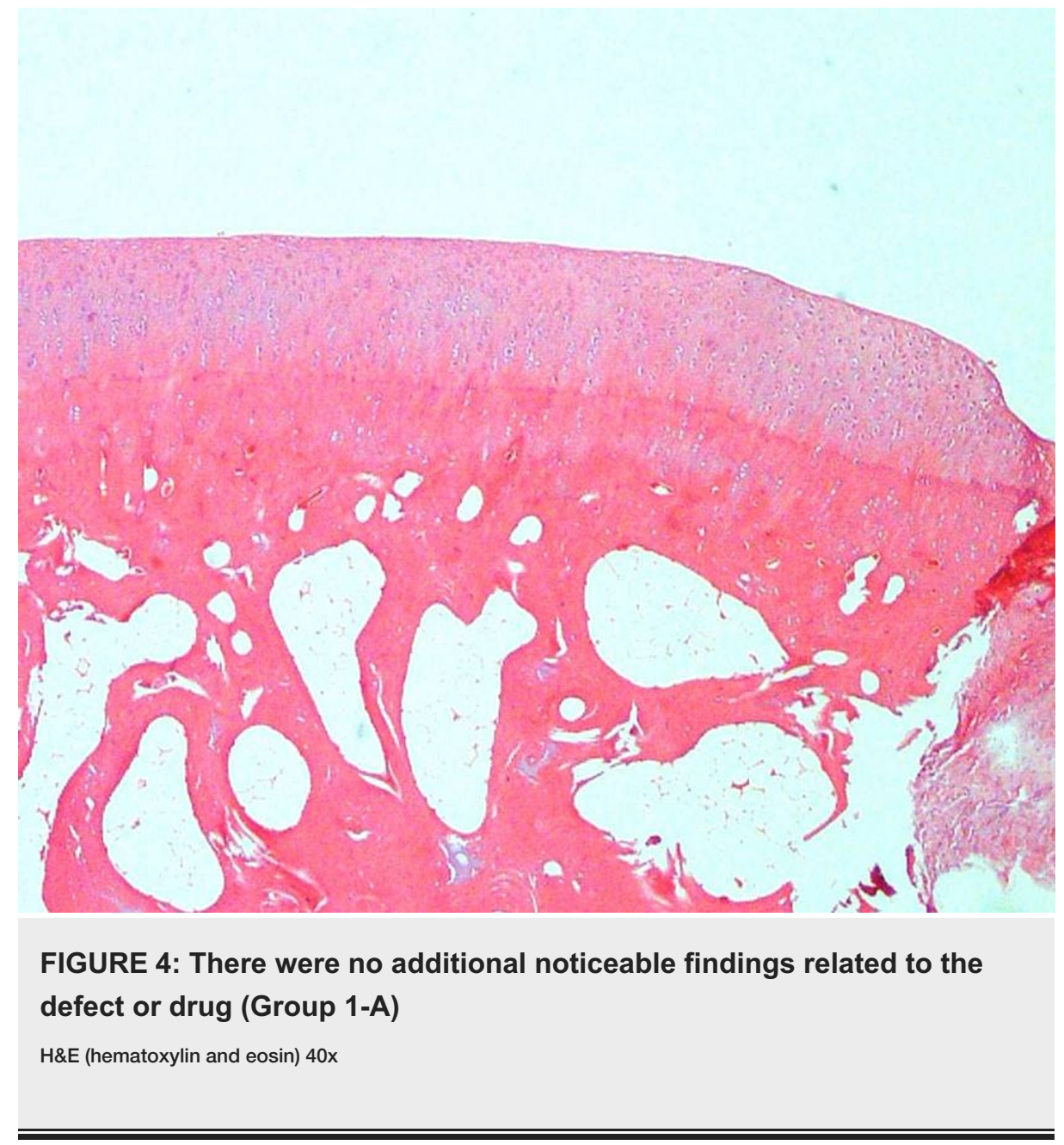

All ICRS II parameters were higher in the saline solution group than in the TXA group in the microfracture group, but no significant difference was observed in the TXA group in the microfracture group except for inflammation, which was similar in the TXA and saline solution groups in the microfracture group (Table 2; Figures 5-8. 


\section{Cureus}

\begin{tabular}{|l|llll|l|}
\hline Parameters & Tranexamic Acid Group (Group 2-A) & \multicolumn{2}{l|}{ Saline Solution Group (Group 2-B) } \\
\hline & Mean \pm SD & Min-Max & Mean \pm SD & Min-Max & p value \\
\hline Tissue morphology & $42.5 \pm 15$ & $30-60$ & $46.6 \pm 16.3$ & $30-70$ & 0.658 \\
Matrix staining & $52.5 \pm 20$ & $30-80$ & $56.6 \pm 32$ & $20-100$ & 0.915 \\
Cell morphology & $76.2 \pm 9.4$ & $70-90$ & $86.6 \pm 12.1$ & $70-100$ & 0.157 \\
Chondrocyte clustering & $60 \pm 32$ & $10-100$ & $66.6 \pm 17$ & $50-100$ & 0.857 \\
Surface architecture & $62.5 \pm 18$ & $50-90$ & $71.6 \pm 22$ & $30-100$ & 0.513 \\
Basal integration & $67.5 \pm 12.5$ & $50-80$ & $70 \pm 25$ & $40-100$ & 0.745 \\
Formation of a tidemark & $41.2 \pm 10.3$ & $30-60$ & $46.6 \pm 20$ & $20-70$ & 0.745 \\
Subchondral bone abnormalities & $61 \pm 21$ & $30-80$ & $61.6 \pm 20$ & $40-90$ & 0.914 \\
Inflammation & 100 & & 100 & & 1 \\
\hline Abnormal calcification/ossification & $87.5 \pm 25$ & $50-100$ & $91.6 \pm 20$ & $50-100$ & 0.759 \\
Vascularization & $37.5 \pm 17$ & $20-60$ & $40 \pm 36$ & $0-100$ & 0.829 \\
\hline Surface/superficial assessment & $67.5 \pm 15$ & $50-80$ & $70 \pm 27$ & $20-100$ & 0.666 \\
Mid/deep zone assessment & $60 \pm 11$ & $50-70$ & $65 \pm 17$ & $40-80$ & 0.511 \\
Overall assessment & $60 \pm 14$ & $40-70$ & $65 \pm 22$ & $30-90$ & 0.591 \\
\hline
\end{tabular}

TABLE 2: Comparison of ICRS II scoring between the microfracture group

ICRS: International Cartilage Repair Society 


\section{Cureus}

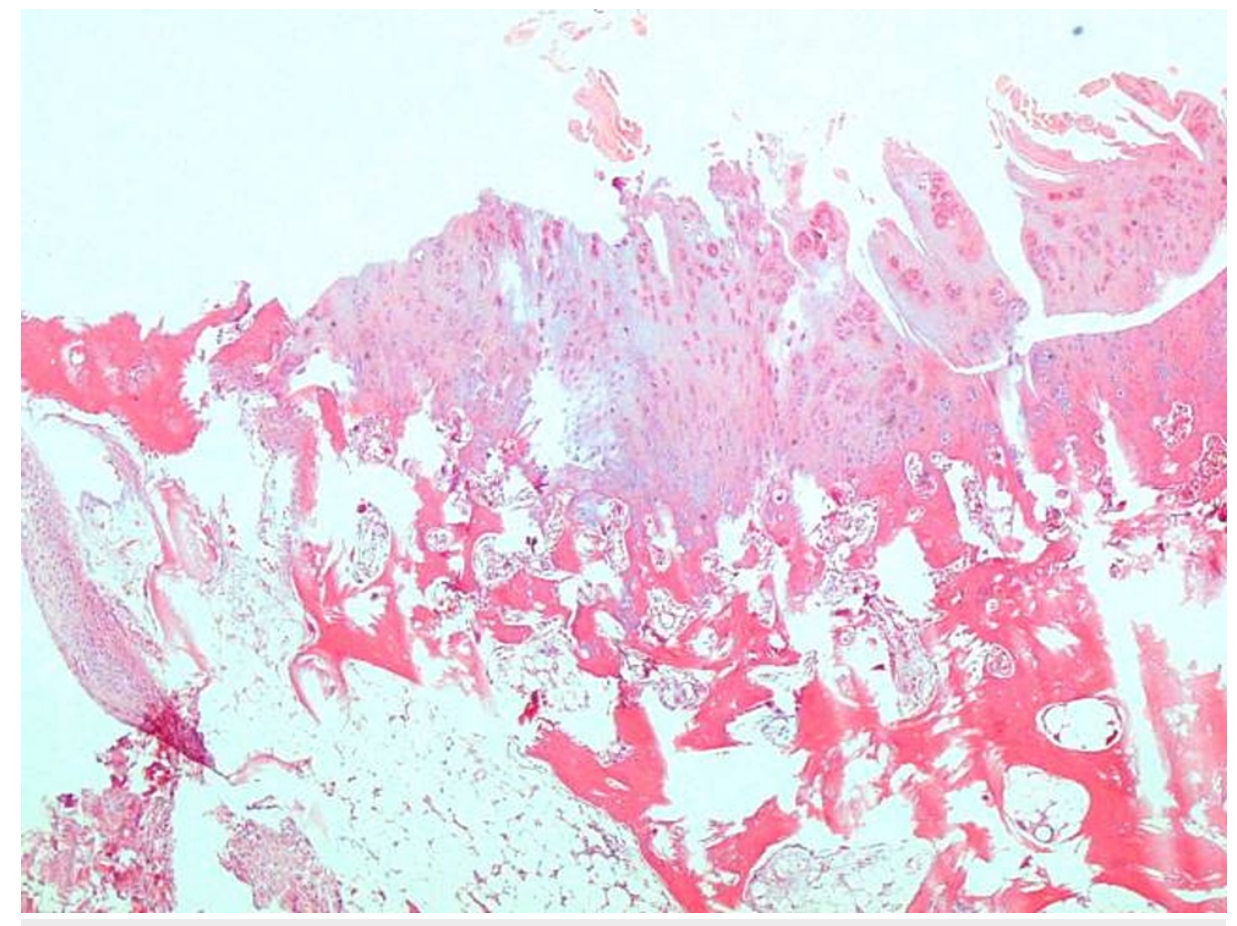

FIGURE 5: Major irregularity, loosening, and disruptions were seen on the surface architecture. Integration with the subjacent bone was poor. Abnormal ossification and chondrocyte clustering (arrows) were also noted (Group 2-B)

H\&E (hematoxylin and eosin) 40x, 100x 


\section{Cureus}

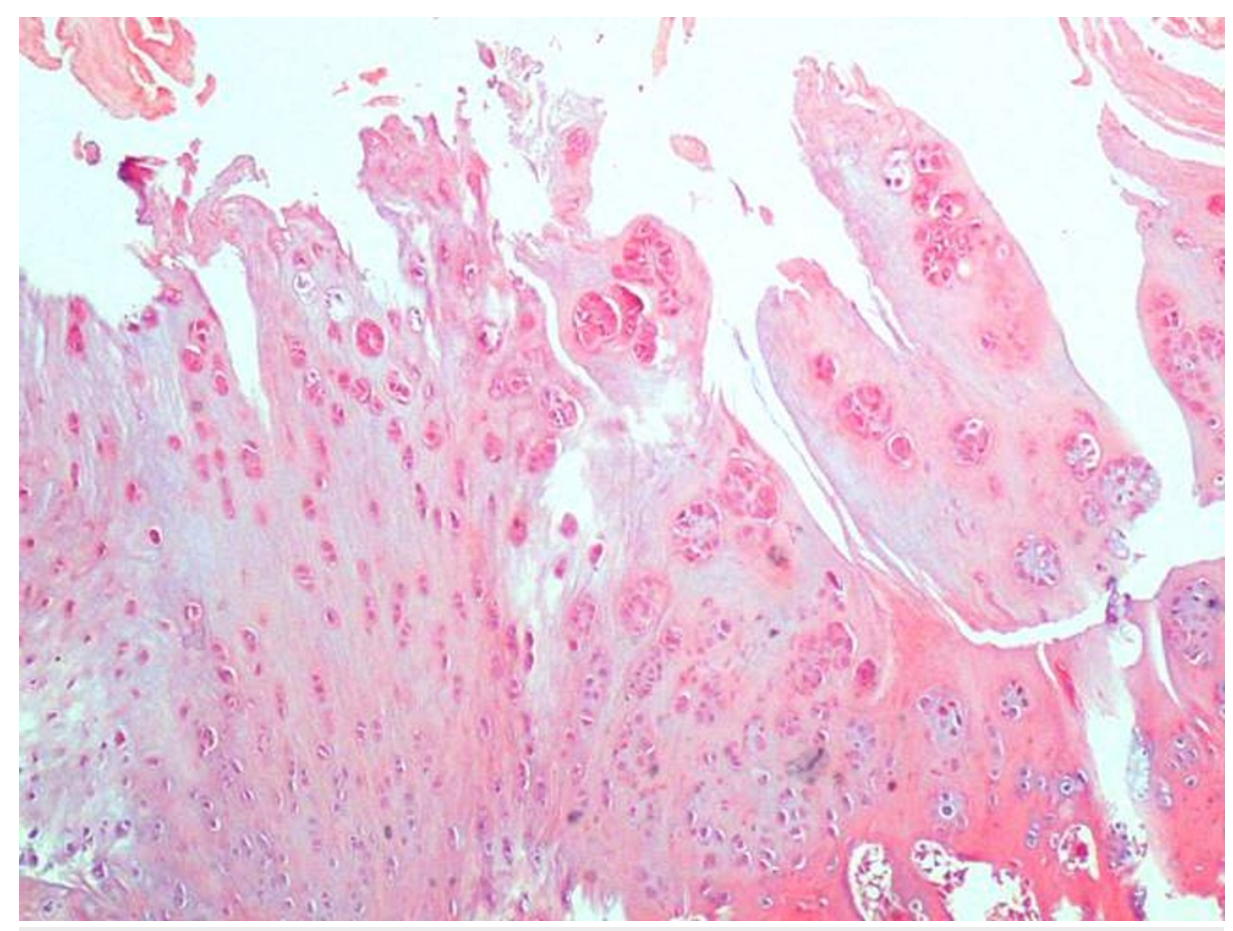

FIGURE 6: Major irregularity, loosening, and disruptions were seen on the surface architecture. Integration with the subjacent bone was poor. Abnormal ossification and chondrocyte clustering (arrows) were also noted (Group 2-B)

H\&E (hematoxylin and eosin) 40x, 100x 


\section{Cureus}

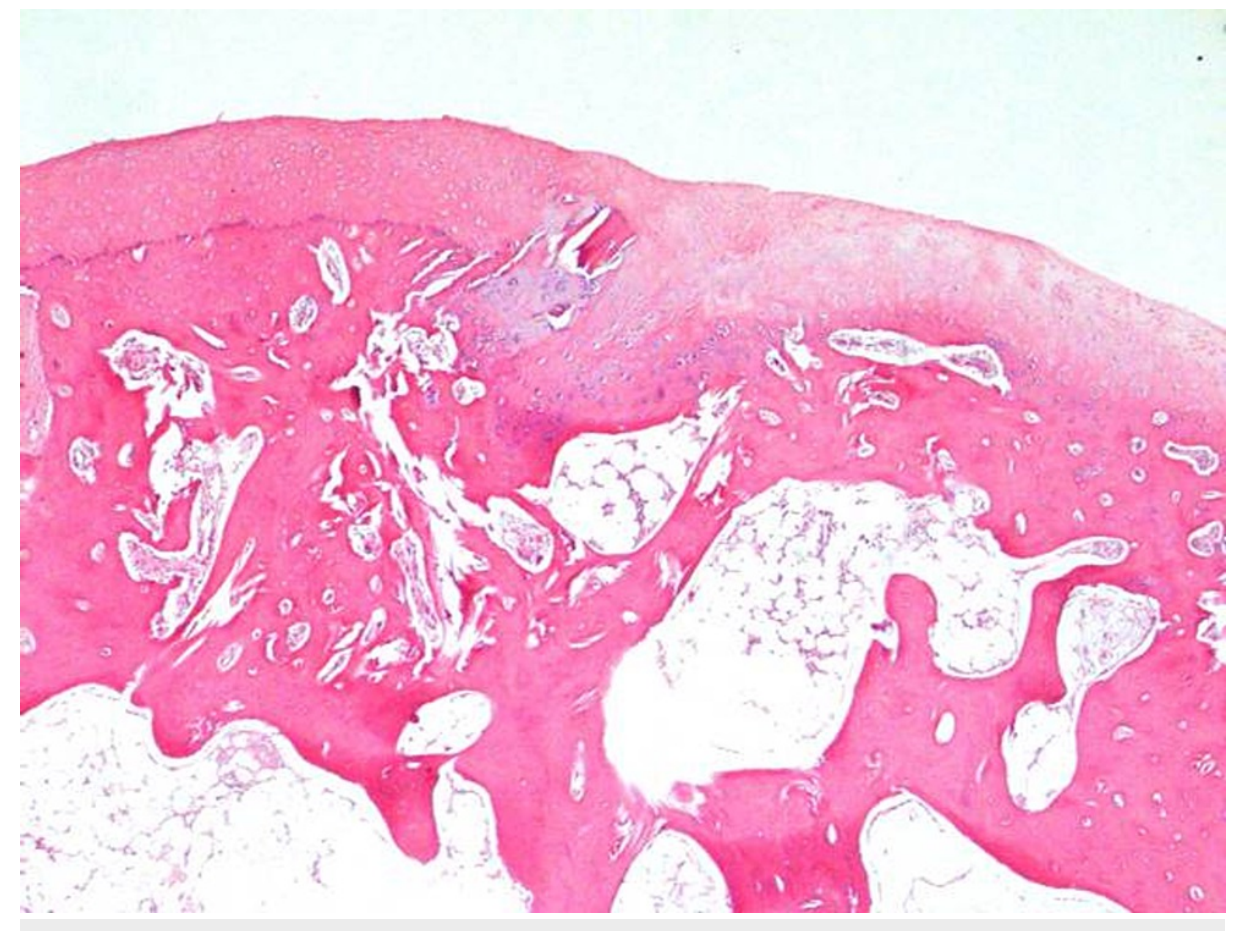

FIGURE 7: Smooth surface architecture and almost full basal integration along the whole cartilage bone interface were observed. The cells associated with the hyaline cartilage were composed of mostly round/oval cells. The tidemark formation between the radial zone and the calcified cartilage was noted (arrows) (Group 2-A). 


\section{Cureus}

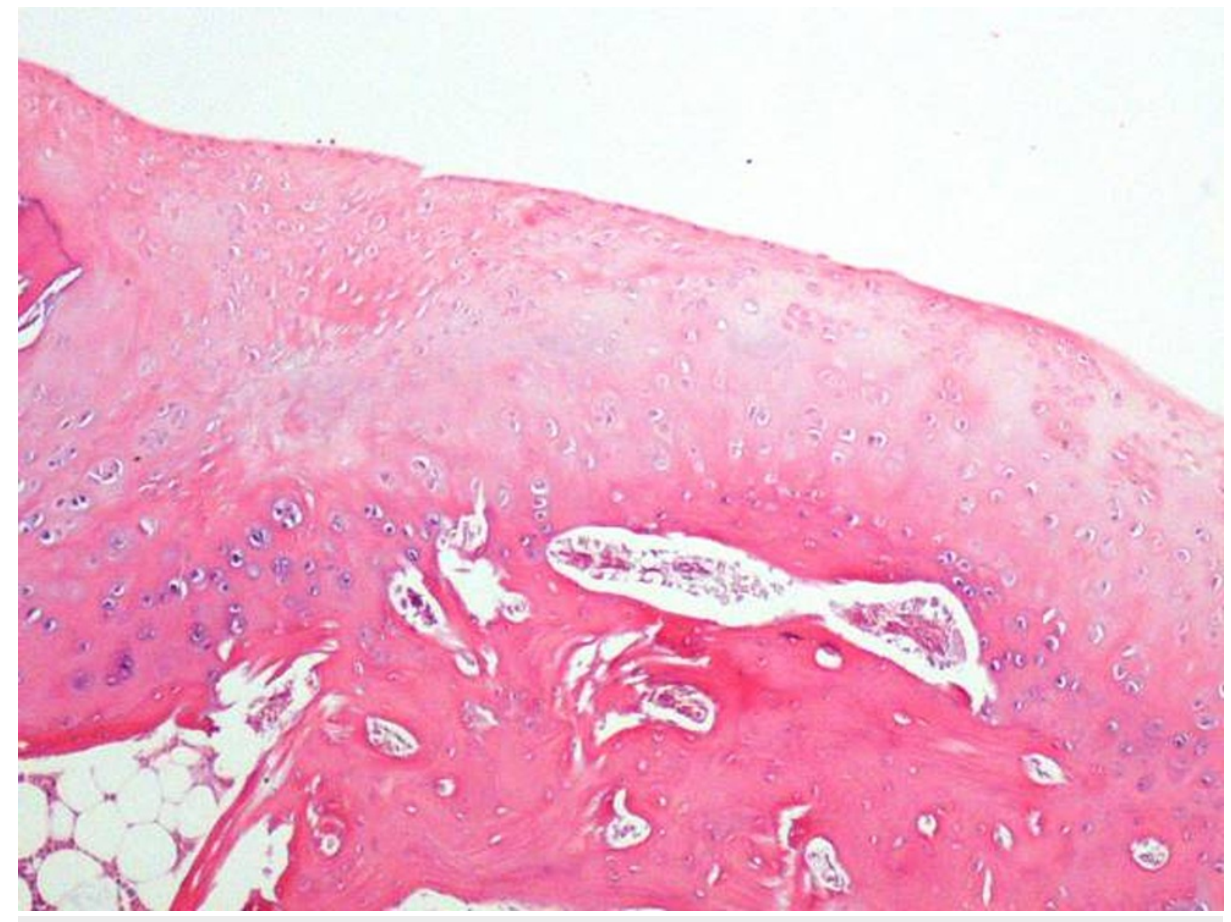

FIGURE 8: Smooth surface architecture and almost full basal integration along the whole cartilage-bone interface were observed. The cells associated with the hyaline cartilage were composed of mostly round/oval cells. The tidemark formation between the radial zone and the calcified cartilage was noted (arrows) (Group 2-A).

H\&E (hematoxylin and eosin) 40x, 100x

Since the organization and quality of regenerative cartilage tissue formed after a microfracture may differ between groups, the difference in appearance in the cross-section is due to this. Basically, the histopathological formation was similar in the two samples.

\section{Discussion}

Although the intra-articular use of TXA is thought to have a less systemic effect, its effect on articular cartilage continues to be investigated. In this study, we investigated the effect of TXA on intact cartilage and the recovery of experimental osteochondral lesions following microfracture in a rabbit model. Based on the results, TXA was observed to have no significant side effects either on intact cartilage tissue and the recovery of experimental osteochondral lesions following microfracture based on the ICRS II scoring system.

One of the primary goals of the previous studies was to investigate the effect of topical TXA on native cartilage. Tuttle et al. investigated the in vitro effects of TXA on bovine cartilage and murine chondrocytes [9]. In that study, they administered TXA in three doses $(25,50$, and $100 \mathrm{mg} / \mathrm{mL})$ and found that the 50 $\mathrm{mg} / \mathrm{mL}$ and $100 \mathrm{mg} / \mathrm{mL}$ doses of TXA had a cytotoxic effect on the chondrocytes and damaged the cartilage. However, a $25 \mathrm{mg} / \mathrm{mL}$ dose of TXA may be an effective and safe dose for the intra-articular use of TXA in native joints. In another study, Parker et al. investigated the side effects of TXA on chondrocytes and determined a safe dose for its intra-articular use in clinical practice [10]. In that study, they found that no cytotoxic effect was observed when in vitro chondrocytes were exposed to TXA in a concentration that could be expected following intravenous administration, whereas cytotoxicity was observed in vitro when the chondrocytes were exposed to a higher concentration following intra-articular administration. In the present study, we investigated the effect of TXA on either intact cartilage and the recovery of osteochondral defects by comparing the saline solution infusion that was evaluated based on the ICRS II scoring system. Furthermore, it is found that the administration of intra-articular TXA did not have a detrimental effect different from saline infusion on the architecture of articular cartilage.

The other main subject is the effect of intra-articular TXA on the recovery of experimentally induced osteochondral defects. Avki et al. investigated the effects of TXA on the recovery of full-thickness articular cartilage defects, wherein $2 \mathrm{~mm}$ diameter osteochondral defects were made in the rabbits and intra-articular TXA was administered intramuscularly every other day for two to four weeks [13]. In that study, they found that TXA treatment had no side effects on the final quality of osteochondral reparative tissue but may 
shorten the modulation to fibrocartilage tissue. In another study, Degirmenci et al. evaluated the effect of intra-articular TXA application on the recovery of experimentally induced osteochondral defects in rabbits [14]. In that study, the rabbits were divided into two groups: Group 1 (study group) and Group 2 (control group). One ml TXA was injected into the knee joints of the study group, and histological evaluations were performed in all samples based on the Brittberg and O'Driscoll scores. Moreover, they found that the administration of TXA improves the recovery time and tissue stability in osteochondral defects. In the present study, we compared the effects of TXA and saline solution on the recovery of experimentally induced osteochondral defects and found that the saline solution group had higher ICRS II scores but was not significantly different.

One strength of this study is that it is an in vivo study, as most of the previous studies have performed an in vitro cell culture study that evaluated the effects of TXA on cartilage or chondrocytes. Therefore, our study is more useful in clinical practice. Another strength is that we compared the effect of TXA and saline solution on either intact cartilage and the recovery of experimentally induced osteochondral defects. Lastly, this study used the ICRS II scoring system, which was developed for assessing cartilage repair tissue and not used in previous studies on the effect of TXA on cartilage and chondrocytes.

Nonetheless, our study had several limitations. First, the sample size was relatively small. Radiological evaluation was not performed especially in the microfracture group. The use of a $10 \mathrm{mg} / \mathrm{kg}$ dose of TXA may be insufficient. In addition, only one scoring system was used for the evaluation of the effect of TXA on cartilage. Last, only one pathologist evaluated the specimens so no interobserver assessment was performed.

\section{Conclusions}

Tranexamic acid widely is used in orthopedic practice, expecting beneficial effects. In our study, we performed an in vivo study evaluating the effect of tranexamic acid on cartilage transformation and proliferation, which was not investigated using the International Cartilage Repair Society II scoring system in the literature. We found that intraarticular tranexamic acid administration in a limited dose did not have a negative impact on healthy cartilage tissue. Finally, we found that intraarticular tranexamic acid administration in a low dose did not have a negative impact on cartilage transformation and proliferation as compared to saline infusion.

\section{Additional Information \\ Disclosures}

Human subjects: All authors have confirmed that this study did not involve human participants or tissue. Animal subjects: Istanbul University Aziz Sancar Institute of Experimental Medicine Laboratories (Istanbul, Turkey) Issued protocol number 2917/135. Conflicts of interest: In compliance with the ICMJE uniform disclosure form, all authors declare the following: Payment/services info: This study was funded by Scientific Research Projects Coordination Unit of Istanbul University: Project number: TSA-2017-20385. Financial relationships: All authors have declared that they have no financial relationships at present or within the previous three years with any organizations that might have an interest in the submitted work. Other relationships: All authors have declared that there are no other relationships or activities that could appear to have influenced the submitted work.

\section{Acknowledgements}

We thank Prof. Dr. Önder Yazıcıoğlu for his help during the study process. This study was conducted at Istanbul University Istanbul Faculty of Medicine, Department of Laboratory Animals Science.

\section{References}

1. Nilsson IM: Clinical pharmacology of aminocaproic and tranexamic acids . J Clin Pathol Suppl (R Coll Pathol). 1980, 14:41-7.

2. Karaaslan F, Karaoğlu S, Mermerkaya MU, Baktir A: Reducing blood loss in simultaneous bilateral total knee arthroplasty: combined intravenous-intra-articular tranexamic acid administration. A prospective randomized controlled trial. Knee. 2015, 22:131-5. 10.1016/j.knee.2014.12.002

3. Charoencholvanich K, Siriwattanasakul P: Tranexamic acid reduces blood loss and blood transfusion after TKA: a prospective randomized controlled trial. Clin Orthop Relat Res. 2011, 469:2874-80. 10.1007/s11999. 011-1874-2

4. Xu X, Xie L, Yu H, Hu Y: Safety and efficacy of tranexamic acid with epinephrine for prevention of blood loss following surgery for trochanteric femoral fractures. Acta Orthop Traumatol Turc. 2020, 54:132-7. 10.5152/j.aott.2020.02.135

5. Zhang Y, Zhang L, Ma X, Jia Y, Wang H, Zhu Y, Liu Y: What is the optimal approach for tranexamic acid application in patients with unilateral total hip arthroplasty?. Orthopade. 2016, 45:616-21. 10.1007/s00132016-3252-y

6. Nugent M, May JH, Parker JD, et al.: Does tranexamic acid reduce knee swelling and improve early function following arthroscopic meniscectomy? A double-blind randomized controlled trial. Orthop J Sports Med. 2019, 7:1-7. 10.1177/2325967119866122 


\section{Cureus}

7. Murkin JM, Falter F, Granton J, Young B, Burt C, Chu M: High-dose tranexamic acid is associated with nonischemic clinical seizures in cardiac surgical patients. Anesth Analg. 2010, 110:350-3.

10.1213/ANE.0b013e3181c92b23

8. Ker K, Prieto-Merino D, Roberts I: Systematic review, meta-analysis and meta-regression of the effect of tranexamic acid on surgical blood loss. Br J Surg. 2013, 100:1271-9. 10.1002/bjs.9193

9. Parker JD, Lim KS, Kieser DC, Woodfield TBF, Hooper GJ: Is tranexamic acid toxic to articular cartilage when administered topically? What is the safe dose?. Bone Joint J. 2018, 100-B:404-12. 10.1302/0301620X.100B3.BJJ-2017-1135.R1

10. Tuttle JR, Feltman PR, Ritterman SA, Ehrlich MG: Effects of tranexamic acid cytotoxicity on in vitro chondrocytes. Am J Orthop (Belle Mead NJ). 2015, 44:E497-502.

11. Standard Guide for in Vivo Assessment of Implantable Devices Intended to Repair Or Regenerate Articular Cartilage. ASTM International, West Conshohocken, PA; 2010.

12. Mainil-Varlet P, Van Damme B, Nesic D, Knutsen G, Kandel R, Roberts S: A new histology scoring system for the assessment of the quality of human cartilage repair: ICRS II. Am J Sports Med. 2010, 38:880-90. $10.1177 / 0363546509359068$

13. Avki S, Hatipoglu F, Yigitarslan K: Evaluation of repair process of osteochondral defects in rabbit articular cartilage with an inhibitor of plasminogen activator (tranexamic acid). Revue Med Vet. 2003, 154:421-5.

14. Degirmenci E, Ozturan KE, Sahin AA, Yilmaz F, Kaya YE: Effects of tranexamic acid on the recovery of osteochondral defects treated by microfracture and acellular matrix scaffold: an experimental study. J Orthop Surg Res. 2019, 14:105. 10.1186/s13018-019-1144-7 